\section{Do Patients Get Whacked When Hospitals Get HACed?}

To the Editor-The Hospital-Associated Condition (HAC) Reduction Program (HACRP) was authorized by the Affordable Care Act requiring Centers for Medicaid and Medicare Services to reduce Medicare Inpatient Prospective Payment System provisions by $1 \%$ for hospitals in the lowest quartile of HAC performance. The program began in Fiscal Year 2015 with discharges beginning October 1, 2014. The HAC reductions are applied after value-based purchasing and readmission penalties. Additionally, the Centers for Medicaid and Medicare Services began reporting HACRP outcomes on the Hospital Compare website in December 2014. The Fiscal Year 2016 HACRP resulted in an estimated \$364 million reduction in hospital payments. ${ }^{1}$ Not surprisingly, this program has profound financial and public relations implications for hospitals. For Fiscal Year 2016, the following hospitalacquired infection outcome indicators are included in the HACRP scoring system: cultures suggestive of central line-associated bloodstream infections or catheter-associated urinary tract infections; surgical site infections after colon surgery or hysterectomies; postoperative sepsis; and lab results suggestive of Clostridium difficile infections or methicillin-resistant Staphylococcus aureus infections. Although most of the HAC score is determined by these infectious outcomes, some noninfectious conditions are included, such as postoperative hip fractures.

Robust efforts have been underway locally, regionally, and nationally to mitigate the risk of hospital-acquired infections. One would expect that the financial impact of HACRP would further incentivize hospitals to do more to reduce such adverse outcomes. However, many hospitals have shifted resources from reducing risk of hospital-acquired infections to reducing the hospital's HAC score by focusing efforts on improving specimen procurement or creating disincentives for submitting specimens to the microbiology laboratory. A total of $5 \%$ to $10 \%$ of adult patients, and a greater fraction of pediatric patients, are colonized with $C$. difficile at the time of hospital admission. If such colonized patients are given a laxative and have a loose stool, their healthcare provider may inadvertently order a test for C. difficile. If that test comes back positive, the positive test result is added to the hospital's HAC score calculation despite the fact that the patient entered the hospital colonized with $C$. difficile and did not have diarrhea that was caused by a $C$. difficile infection. In other cases, $C$. difficile testing is ordered on a patient with a fever but without diarrhea and if the test comes back positive, that result too is added to the hospital's HAC score calculation. Rather than focusing efforts on reducing a patient's risk of acquiring $C$. difficile in the hospital from contaminated equipment and environmental surfaces or from unclean hands, or reducing exposure to antibiotics most associated with C. difficile infection in such colonized patients, some hospitals have shifted resources to assure proper procurement of stool for C. difficile testing in an effort to reduce the HAC score.

Patients with bladder catheters all develop microbial colonization of the bladder if these catheters are kept in too long. If urine is collected in a patient with such a catheter but without any symptoms of a urinary tract infection, the urine is often contaminated and that result will be added to the hospital's HAC score calculation erroneously classified as a urinary tract infection. Some hospitals have focused their efforts on reducing the ordering of urine cultures in such catheterized patients unless clinically indicated. This intervention reduces the hospital's HAC score but it does not reduce the patient's risk of developing a bladder catheter-associated urinary tract infection. The latter requires a culture change that focuses on reducing unnecessary bladder catheter insertion and limits the duration of catheterization in those who have a bladder catheter. Making sure that specimens are sent to the microbiology lab only when clinically indicated will reduce indiscriminate antibiotic use by reducing the likelihood that physicians will administer antibiotics in patients who are asymptomatically colonized with various bacteria, for whom such antibiotic exposure is unwarranted and potentially harmful. Such antibiotic stewardship is obviously vitally important; however, if there is a shift of limited resources from basic infection prevention activities to specimen procurement, this may increase the risk of acquiring healthcare-associated infections.

The idea of financially motivating hospitals to reduce infection rates is a laudable paradigm shift. Though this was the intent of the HACRP, a potential unintended consequence is a hospital's pursuit of what is viewed as low-hanging fruit, namely improving specimen procurement, thereby reducing the HAC score by reducing the number of specimens inadvertently sent to the microbiology lab, or worse, creating other disincentives to sending specimens when clinically indicated. Such interventions don't reduce a hospitalized patient's risk of getting a methicillin-resistant $S$. aureus or C. difficile infection, or a surgical site, bloodstream, or urinary tract infection. We need hospitals to thread both needles without robbing Peter to pay Paul. Improving the quality of care and reducing patient harm are of paramount importance. Having the necessary resources (ie, personnel, expertise, information technology support) to do so is an expectation that our patients deserve.

\section{ACKNOWLEDGMENTS}

Financial support. None reported.

Potential conflicts of interest. The author reports no conflicts of interest relevant to this article.

Leonard A. Mermel, DO, ScM, AM (Hon), FSHEA, FIDSA, FACP

Affiliations: Alpert Medical School of Brown University, Providence, Rhode Island; and Department of Epidemiology and Infection Control, Rhode Island Hospital, Providence, Rhode Island. 
Address correspondence to Leonard A. Mermel, DO, Department of Epidemiology and Infection Control, Rhode Island Hospital, 593 Eddy St, Providence, RI 02903 (lmermel@lifespan.org).

Infect Control Hosp Epidemiol 2017;38:249-250

(C) 2016 by The Society for Healthcare Epidemiology of America. All rights reserved. 0899-823X/2017/3802-0021. DOI: 10.1017/ice.2016.267

\section{REFERENCE}

1. Centers for Medicare and Medicaid Services (CMS). Fiscal year (FY) 2016 results for the CMS hospital-acquired conditions (HAC) reduction program. CMS website. https://www.cms.gov/ Newsroom/MediaReleaseDatabase/Fact-sheets/2015-Fact-sheetsitems/2015-12-10-2.html. Published December 10, 2015. Accessed November 6, 2016.

\section{Postdischarge Surveillance: Value and Problems Perceived by Infection Control Practitioners in Switzerland}

To the Editor-Switzerland is one of the few countries where routine postdischarge surveillance (PDS) for the surveillance of surgical site infections (SSIs) is practiced by telephone interview 1 month (and for implanted devices a second interview at 12 months) after the procedure, which is comparable with the system in the Netherlands. ${ }^{1}$

This survey was designed to analyze the perceptions on work load and value of PDS by Swiss infection control practitioners in order to assess the efficiency of resource utilization. The online questionnaire was distributed in December 2015 and January 2016. A major limitation of the study is the subjective assessment method of the survey, but the high response rate of $76(62.3 \%)$ of the 122 Swiss hospitals that were asked to participate provides a representative sample.

Although the practical value of PDS related to clinical infection control is rated moderate on an 8-item Likert scale, the work load is rated high compared with other duties (Figure 1). A total of 23 (37.1\%) of the 62 respondents for this item say that they definitely have curtailed other duties owing to the requirements of PDS and 13 (20.9\%) feel that sometimes they neglect other duties because time is needed for PDS. A total of 30 respondents $(48.4 \%)$ would define the costs and effort for PDS for the hospital as high but 34 (55.8\%) agree that without PDS many SSIs would not be detected. The time effort for one telephone interview and data logging was

8

7

6

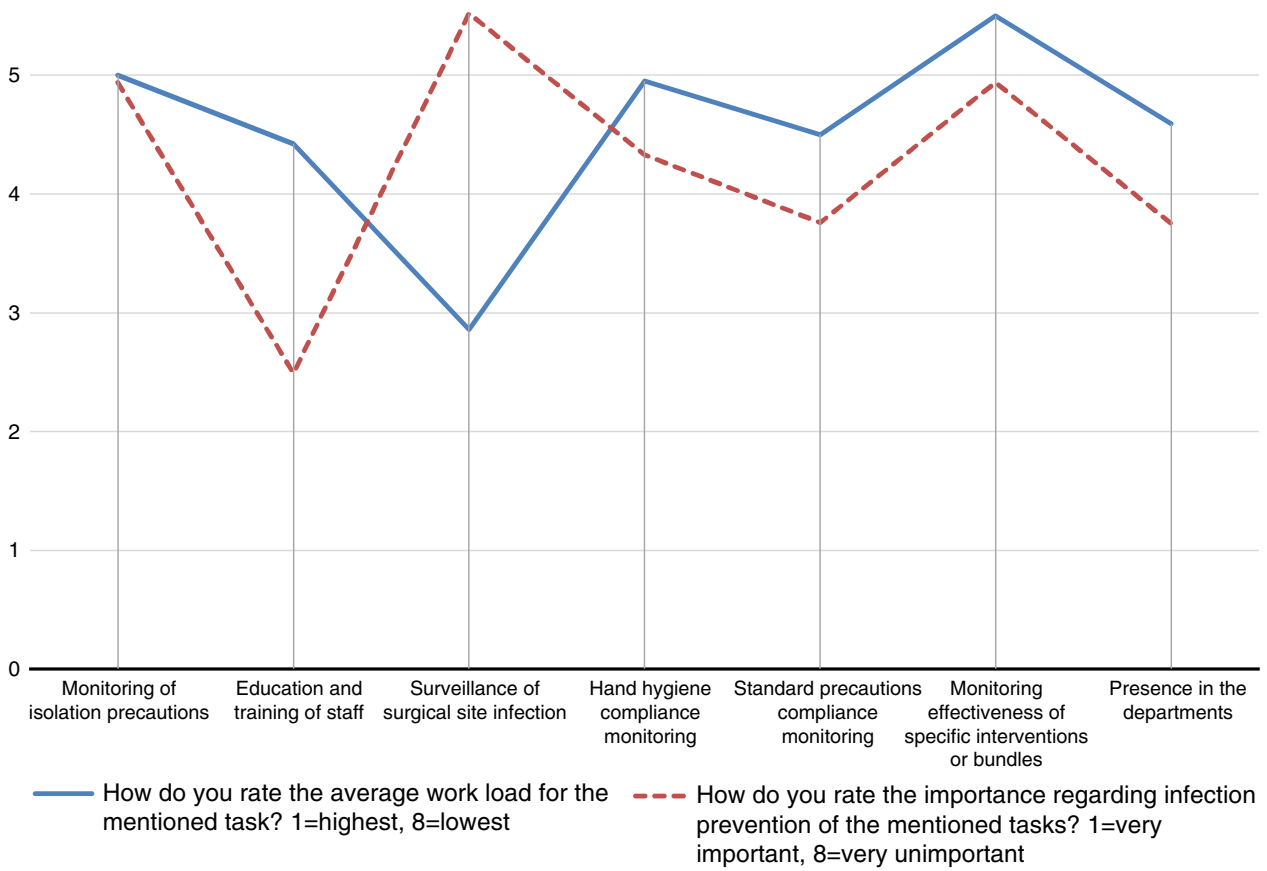

FIGURE 1. Comparative overview of reported workload effort and perceived importance of typical tasks of Swiss infection control practitioners. 